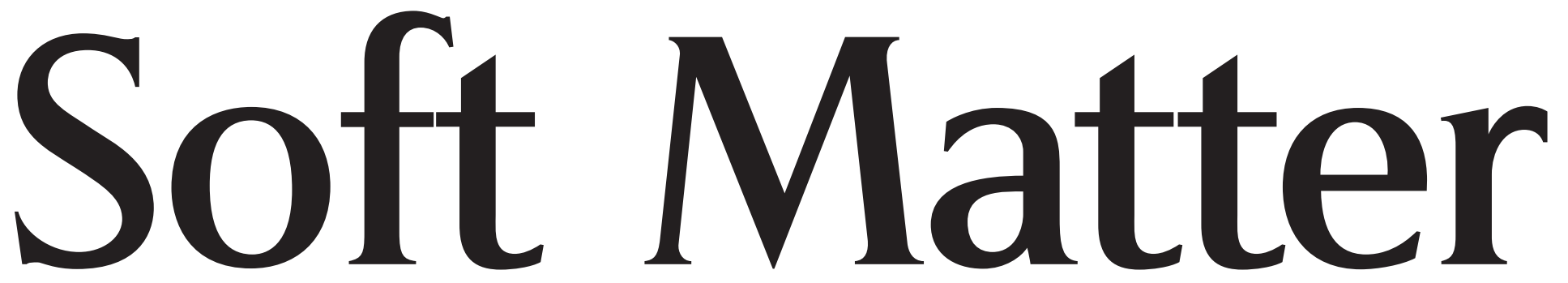


Cite this: Soft Matter, 2013, 9, 10705

\title{
Interbilayer repulsion forces between tension-free lipid bilayers from simulation $\uparrow$
}

\author{
Y. G. Smirnova, ${ }^{\text {a }}$ S. Aeffner, ${ }^{\text {b }}$ H. J. Risselada, ${ }^{c}$ T. Salditt, $^{\text {b }}$ S. J. Marrink, ${ }^{d}$ M. Müller ${ }^{a}$ \\ and V. Knecht ${ }^{\text {ef }}$
}

Here we report studies on biologically important intermembrane repulsion forces using molecular dynamics (MD) simulations and experimental (osmotic stress) investigations of repulsion forces between 1-palmitoyl-2-oleyl-sn-glycero-3-phosphocholine bilayers. We show that the repulsion between tensionfree membranes can be determined from MD simulations by either (i) simulating membrane stacks under different hydration conditions (unrestrained setup) and monitoring the change in the area per lipid upon dehydration or (ii) simulating two single punctured membranes immersed in a water reservoir and controlling the center-of-mass distance between the bilayers using an external potential (umbrella sampling setup). Despite the coarse-grained nature of the (MARTINI) model employed, the disjoining pressure profiles obtained from the simulations are in good agreement with our experiments. Remarkably, the two setups behave very differently in terms of membrane structure, as explained by considerations using elasticity theory, and the balance of interactions. In the unrestrained setup, dehydration decreases the area per lipid and lipid entropy. Dehydration in the umbrella sampling setup, in contrast, leads to an increase in area per lipid and lipid entropy. Hence, in the latter case, entropic effects from protrusion and zippering forces appear to be overcompensated by the entropy gain due to the disorder emerging from the expansion of the bilayers. The balance of interactions involves near cancellation of large opposing terms, for which also intramembrane and water-water interactions are important, and which appears to be largely a consequence, rather than the cause, of the intermembrane repulsion. Hence, care must be taken when drawing conclusions on the origin of intermembrane repulsion from thermodynamic analyses.

Received 27th June 2013

Accepted 6th August 2013

DOI: $10.1039 / \mathrm{c} 3 \mathrm{sm} 51771 \mathrm{c}$

www.rsc.org/softmatter the equilibrium spacing between the membranes composed of neutral phospholipids is in the range of 2-3 nm arising from a balance between short-range repulsion and long-range van-derWaals attraction. Dehydration of the intermembrane contact is likely involved in fusion. ${ }^{4}$ At low hydration when the water layer thickness between bilayers is less than $2 \mathrm{~nm}$ the so-called "hydration force" causes strong repulsion between bilayers. ${ }^{5}$ For lipid bilayers repulsion forces have been measured experimentally using the osmotic stress method $^{6}$ or the surface forces apparatus (SFA). ${ }^{7}$ The experiments yielded a measure of the pressure-distance or the force-distance relationship between interacting phospholipid bilayers, and it was shown that repulsion can be numerically described as a pressure that decays exponentially with the bilayer separation distance, characterized by a pre-exponential coefficient and a decay distance. The typical decay distance of phospholipid bilayers was found to be $0.2-0.3 \mathrm{~nm} .^{8}$ Attempts have been made to calculate the hydration force using continuum theory. ${ }^{\mathbf{9 - 1 1}}$ According to early formulations, the pre-exponential coefficient reflects the degree of ordering of the boundary water by the surface and the decay distance is related to the propagation of the ordering through the water. Ultimately, for large separation

\footnotetext{
${ }^{a}$ Georg August University, Institute for Theoretical Physics, 37077 Göttingen, Germany E-mail: smirnova@theorie.physik.uni-goettingen.de

${ }^{b}$ Georg August University, Institute for X-Ray Physics, 37077 Göttingen, Germany ${ }^{c}$ Max-Planck Institute for Biophysical Chemistry, 37077 Göttingen, Germany ${ }^{d}$ Groningen Biomolecular Sciences and Biotechnology Institute, University of Groningen, Nijenborgh 4, 9747 AG Groningen, The Netherlands

${ }^{e}$ Max-Planck Institute of Colloids and Interfaces, Department of Theory and BioSystems, Research Campus Golm, 14424 Potsdam, Germany

${ }^{f}$ Albert Ludwigs University, Institute of Physics, 79104 Freiburg, Germany

$\dagger$ Electronic supplementary information (ESI) available. See DOI: $10.1039 / \mathrm{c} 3 \mathrm{sm} 51771 \mathrm{c}$
} 
this length scale is set by the correlation length of water in the bulk and, hence, it is independent of the membrane properties. Different magnitudes of forces were attributed to different degrees of surface polarization and the common decay length was attributed to the size of water molecules. ${ }^{10}$ Later it was shown that lipid protrusions contribute to the intermembrane repulsion forces at a similar length scale to the hydration forces leading to the exponentially decaying disjoining pressure with the temperature-dependent decay length, $\lambda_{t}$, for a fixed lipidsolvent mixture. ${ }^{12,13}$ The SFA experiments probe primarily the contribution from the hydration forces as here the bilayers are rigid and immobilized on mica surfaces, whereas the osmotic stress experiments performed with fluid membranes probe contributions from both hydration forces and lipid protrusions. $^{2}$

While experiments and continuum theory quantitatively and qualitatively evaluate the interbilayer forces, respectively, they cannot describe the molecular origin of these forces, i.e. correlate the measured repulsion force with the properties of a specific physical system. Molecular dynamics (MD) simulations ${ }^{\mathbf{1 4 - 1 9}}$ can help to understand the interbilayer forces on the molecular level.

Previously, in order to calculate hydration repulsion, simulations were performed either in the grand canonical ensemble ${ }^{15,16}$ to calculate the hydration repulsion between supported membranes or in the canonical ensemble with finite objects around which water can flow, ${ }^{17,18}$ such that the chemical potential of water between the membranes was fixed and equal to the bulk value. Both approaches show the dependence of the pressure or the free energy on the separation distance between membranes or finite objects, respectively. Atomistic simulations of two graphene plates decorated with phosphatidylcholine head groups indicated that the reduction in the favorable interactions between head groups and water molecules, especially due to the breaking of strong hydrogen bonds between phosphates and water molecules, is responsible for the hydration repulsion. ${ }^{17,18}$

Gentilcore et al. ${ }^{19}$ treated membranes with a constant area in the canonical (NVT) ensemble in salt solutions. By using the umbrella sampling method the free energy as a function of membrane separation was calculated. However, systematically higher values compared to experiments were obtained. The authors argued that the possible source of error was due to the fixed membrane area employed in their study, as opposed to the experimental situation for osmotic stress studies where the area per lipid decreases with dehydration. ${ }^{20,21}$ Another reason could be that MD simulations overestimate the effect of $\mathrm{NaCl}$ on the membrane charge and thus the salt effect on the repulsion forces. ${ }^{22}$ The atomistic simulations by Gentilcore et al. suggested that the repulsion force is due to the increase of solvent ordering as the bilayers become dehydrated. This observation correlates with decreased lipid diffusion and redistribution of hydrogen bonds between water and lipids.

Recently a new approximation method for estimating interbilayer repulsion from atomistic simulations was proposed..$^{23,24}$ In order to obtain the repulsive pressure from simulations in an NVT ensemble and to compare results with those of experiments where the chemical potential of water is controlled, Schneck et al. introduced a so-called thermodynamic extrapolation method.
The advantage of this approach is that the disjoining pressure between membranes can be efficiently and accurately calculated (with a pressure resolution of $\sim 15$ bar) and directly compared with experimental results. However, in ref. 23 and 24 a constant area and thickness of the membrane at different hydration levels were assumed. As mentioned earlier, however, experiments $\mathbf{s}^{\mathbf{2 0 1 2}, 25}$ and atomistic simulations ${ }^{26}$ show that the bilayer structure changes upon dehydration, i.e. the bilayer thickness increases and the area per lipid decreases at high osmotic pressure or low hydration. Hence, the membrane systems considered in ref. 19 and 24 were most likely under tension.

Here we implement an approach to calculate the repulsion between tension-free membranes ${ }^{27,28}$ using the experimentally more relevant NPT ensemble by which the decrease in the bilayer area with dehydration for osmotic stress experiments is accounted for. We quantitatively compare our results with those of (i) new osmotic stress experiments and (ii) simulations using the umbrella sampling method to control the interbilayer distance, mimicking early processes in SNARE protein-induced membrane fusion.

Another question considers the minimal model properties needed to reproduce the correct behavior of the bilayer disjoining pressure. If solvent ordering and hydrogen bonding underlie hydration repulsion the question arises how well can repulsion forces be reproduced with a solvent model that lacks this aspect of the atomic nature, such as, e.g., the MARTINI coarse-grained model, ${ }^{29}$ where four water molecules are represented by a single interaction site, keeping only translational degrees of freedom. In particular, this model has been used to simulate membrane fusion, ${ }^{\mathbf{3 0}-\mathbf{3 3}}$ where strong repulsion between bilayers is an important issue.

In this article we study the hydration repulsion between 1-palmitoyl-2-oleoyl-sn-phosphatidylcholine (POPC) bilayers in the liquid-crystalline $\left(L_{\alpha}\right)$ phase in water. POPC is the most abundant lipid in animal cell membranes and contains one chain that is fully saturated at the $s n-1$ position and another chain with a single double bond at the $s n-2$ position. We used both high resolution experiments and coarse-grained MD simulations and compared them with atomistic simulations from ref. 26.

\section{Materials and methods}

\section{$\mathrm{X}$-ray reflectivity measurements}

The osmotic stress method combined with X-ray diffraction is a classical technique to study the bilayer interaction, see for example ref. 34 and 35. However, measuring only one bilayer parameter such as the lamellar repeat spacing $d$ as a function of osmotic pressure is not sufficient in general, as discussed for example in ref. 21. To determine the interbilayer repulsion as a function of water layer $d_{\mathrm{w}}$, which is required for quantitative comparison with theory or simulations, the entire electron density profile $\rho(z)$ is needed. The so-called gravimetric method used in many earlier studies is based on the simplifying assumption that lipid and water molecules partition into distinct and well-defined layers and maintain their molecular volumes in the bulk, thus excluding possible intercalation, as well as lipid protrusion at the molecular level. ${ }^{1,36}$ In contrast, the osmotic stress method in 
conjunction with electron density profile (EDP) analysis provides the actual bilayer structure, which changes with the relative humidity (RH). Furthermore, the use of oriented lamellar phases typically yields a significantly higher number of equidistant Bragg peaks indexed by $n=1,2, \ldots$ along the $q_{z}$ axis, than diffraction studies in isotropic solution, increasing the experimental resolution. Finally, the precise distinction between momentum transfer perpendicular $q_{z}$ and $q_{\|}$parallel to the membrane surface allows for obtaining additional information. For example the in-plane structure factor describing the fluid chain-chain correlations $S$ $\left(q_{\|}\right)$can be measured. In the present case, comparing experimental and simulated $S\left(q_{\|}\right)$(data shown in the ESI $\dagger$ ) gives additional evidence that the molecular scales are sufficiently well represented by the MARTINI coarse-grained model.

Experimentally, the total repulsive pressure between lipid bilayers was quantified by EDP analysis at different levels of osmotic stress. ${ }^{\mathbf{1 , 3 5 , 3 7}}$ The applied protocols are described elsewhere in detail. ${ }^{21,38,39}$ In brief, stacks of about 1500 aligned lipid bilayers were prepared on silicon substrates and placed in an environmental chamber with precise $\mathrm{RH}$ control. ${ }^{39}$ The latter allowed us to tune the osmotic pressure $P_{\mathrm{osm}}=-\frac{k_{\mathrm{B}} T}{v_{\mathrm{w}}} \ln (\mathrm{RH} / 100 \%)$, which is similar to exerting a mechanical pressure that pushes lipid bilayers together ${ }^{37}$ and effectively dehydrates the bilayer stack. Here, $k_{\mathrm{B}}=1.38066 \times$ $10^{-23} \mathrm{~J} \mathrm{~K} \mathrm{~K}^{-1}$ denotes Boltzmann's constant, $T$ is the absolute temperature and $v_{\mathrm{w}}=3 \times 10^{-29} \mathrm{~m}^{3}$ is the volume of a single water molecule. Prior to the experiment, the $\mathrm{RH}$ sensor in the vicinity of the sample was calibrated by saturated salt solutions. The sample chamber was mounted on a home-built X-ray reflectometer with $\mathrm{Cu}-\mathrm{K}_{\alpha}$ radiation (wavelength $\lambda=1.541 \AA$ ). The X-ray beam was parallelized and monochromatized by using a Goebel mirror. X-ray reflectivity curves were recorded in $\theta / 2 \theta$ geometry for angles $2 \theta=1-17^{\circ}$ in steps of $\Delta(2 \theta)=0.01^{\circ}$ (Fig. 1a) at different values of $\mathrm{RH}$, thus probing the reflected intensity as a function of momentum transfer $q_{z}=\frac{4 \pi}{\lambda} \sin \theta$ perpendicular to the lipid bilayers at different hydration levels. Starting at $\mathrm{RH} \approx 96 \%$, the relative humidity was successively lowered to $\mathrm{RH} \approx 33 \%$. This corresponds to a pressure range of $P=$ $0.06-1.51 \times 10^{8} \mathrm{~N} \mathrm{~m}^{-2}$. In thermal equilibrium, this pressure must be balanced by the total repulsive pressure between lipid bilayers.

From the reflectivity curves, the lattice constant $d=n \frac{2 \pi}{q_{n}}$ can immediately be obtained from the position $q_{n}$ of the $n^{\text {th }}$ Bragg peak. If the only effect of dehydrating the bilayer stack was removal of water from the interbilayer space, one would expect a monotonous decrease of $d$ while reducing RH. However, we observed that $d$ changes in a nonmonotonous fashion upon dehydration (see also Fig. 1d). A minimum of $d$ was observed at $\mathrm{RH} \approx 83 \%$, upon further dehydration $d$ increased slightly again. As discussed below, this can be explained by an increase in bilayer thickness upon dehydration, which in the case of POPC seems to outweigh the simultaneous decrease in water layer thickness. At $\mathrm{RH} \approx 50 \%$, a sudden increase of $d$ from about $52 \AA(\mathrm{RH}=50 \%)$ to about $59 \AA(\mathrm{RH}=40 \%)$ was observed,
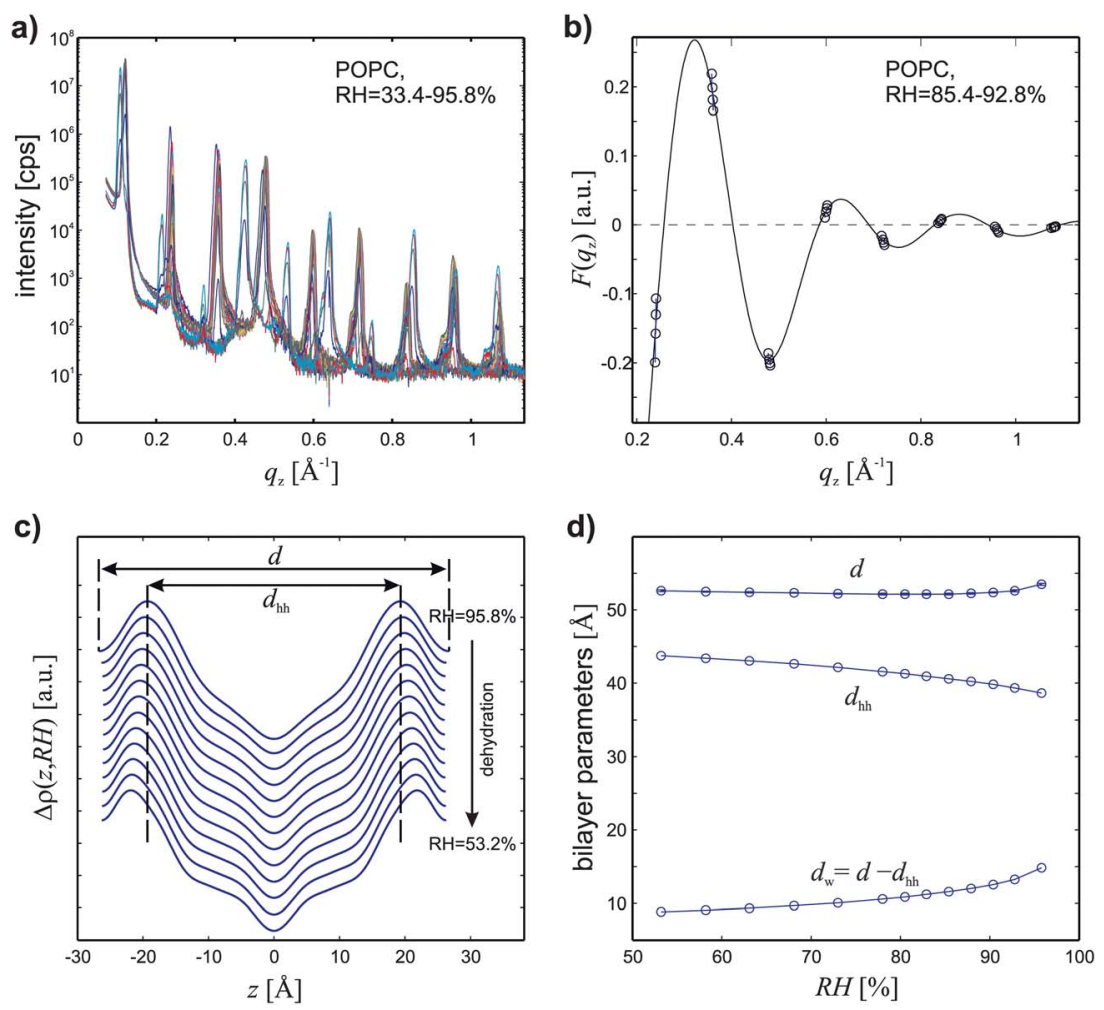

Fig. 1 Experimental results obtained from aligned POPC multi-bilayer stacks by $\mathrm{X}$-ray reflectivity: (a) reflectivity curves at different RH levels. (b) Phase retrieval by the swelling method (data points of $v_{1}\left|F_{1}\right|$ are not shown) (c) reconstructed electron density profiles (EDPs) on an arbitrary scale, shifted vertically for clarity. The bilayer thickness $d_{\mathrm{hh}}$ increases upon dehydration. (d) Structural parameters $d, d_{\mathrm{hh}}$, and $d_{\mathrm{w}}$. 
accompanied by the appearance of up to 15 visible Bragg Peaks (data not shown). We attribute this latter observation to a dehydration-induced phase transition from the liquid-crystalline $L_{\alpha}$ phase to a gel phase with more extended acyl chains and smaller area per lipid headgroup, as also observed in earlier studies by differential scanning calorimetry. ${ }^{40}$ For further analysis of interbilayer forces, only data in the $L_{\alpha}$ phase (i.e. $\mathrm{RH}$ $>50 \%$ ) were used.

The EDP, i.e. the electron density contrast $\Delta \rho(z)$, at each level of hydration in the $L_{\alpha}$ phase was reconstructed on an arbitrary scale by the Fourier cosine series $\Delta \rho(z)=\sum_{n} \nu_{n}\left|F_{n}\right| \cos \left(q_{n} \cdot z\right)$. The form factor amplitudes $\left|F_{n}\right| \propto \sqrt{n \cdot I_{n}}$ were extracted from the integrated Bragg peak intensities $I_{n}$. The phase factors $v_{n}= \pm 1$ (due to centrosymmetry) were obtained with the aid of the swelling method for phase retrieval (e.g. ref. 38 and 41 and references therein) as follows: firstly, due to the nonmonotonous curve $d(\mathrm{RH})$, only data for $\mathrm{RH}>85 \%$ in the regime of "normal" swelling were used (Fig. 1b). The obtained phase combination $\{--+-+-+--\}$ was then applied to the entire range of the $L_{\alpha}$ phase with the following changes: since the bilayer structure and thus the corresponding form factors (i.e. the Fourier components of the electron density) are expected to change continuously in the absence of a phase transition, a sign change of the phase factor $v_{n}$ can only occur at roots of the corresponding $F_{n}$. Starting from the initial phase combination $\left\{v_{n}\right\}$ given above, the signs of some $v_{n}$ were changed if the corresponding $\left|v_{n}\right|$ exhibited a minimum.

In the resulting EDPs (Fig. 1c), the headgroup-headgroup distance $d_{\mathrm{hh}}$ and water layer thickness $d_{\mathrm{w}}=d-d_{\mathrm{hh}}$ as defined by the position of electron density maxima are readily obtained as a function of $\mathrm{RH}$ or, equivalently, pressure (Fig. 1d). $d_{\mathrm{w}}$ decreases monotonously with increasing pressure, while $d_{\mathrm{hh}}$ increases. In summary, both effects almost cancel each other and lead to the nonmonotonous change of $d=d_{\mathrm{hh}}+d_{\mathrm{w}}$. Finally, fitting a decaying exponential $P_{0} \exp \left(-d_{\mathrm{w}} / \lambda_{t}\right)$ yields the disjoining pressure amplitude $P_{0}$ and the decay length $\lambda_{t}$. Importantly, the numerical value of $P_{0}$ depends on the definition of $d_{\mathrm{w}}$ (see below).

\section{Simulation details}

Simulations were performed with the GROMACS software package, version 3.3.2. ${ }^{42}$ The systems considered in this work were studied under periodic boundary conditions using the MARTINI coarse-grained model, ${ }^{29}$ see Fig. 2. Here, on average, four heavy atoms or four water molecules are represented by one coarse-grained bead. Covalent bonds of lipids are modeled by springs, and the stiffness of the lipid tails is provided by angle potentials. The polarity of the groups is modeled by an effective Lennard-Jones (LJ) potential. LJ interactions are truncated at $1.2 \mathrm{~nm}$ and shifted between 0.9 and $1.2 \mathrm{~nm}$. The zwitterionic character of the lipid molecules is modeled by charges on the choline and phosphate groups. These charged groups interact via a Coulomb potential with a relative dielectric constant $\varepsilon=15$ being shifted between 0.9 and $1.2 \mathrm{~nm}$ to mimic the effect of distance dependent screening. A $1.2 \mathrm{~nm}$ cut-off was used for the neighbor list updated every 10 time steps. The effective time step used was 160 fs (the effective time scale is defined via the diffusion of lipid molecules ${ }^{29}$ ). Simulations were conducted at $300 \mathrm{~K}$ by coupling the lipids and water separately to a heat bath using a Berendsen thermostat ${ }^{43}$ with a relaxation time of $0.4 \mathrm{ps}$. The box dimensions normal and lateral to the bilayer were scaled independently to maintain a pressure of 1 bar in each direction corresponding to zero membrane tension using the Berendsen barostat ${ }^{\mathbf{4 3}}$ with a relaxation time of $0.8 \mathrm{ps}$.

First, to study different hydration levels and finite size effects (the number of lipids in the system) several systems with different water and lipid contents were simulated. Two series of simulations were performed, each using 15 systems with

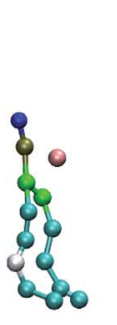

(a)

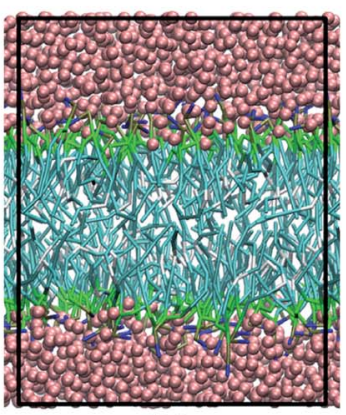

(b)

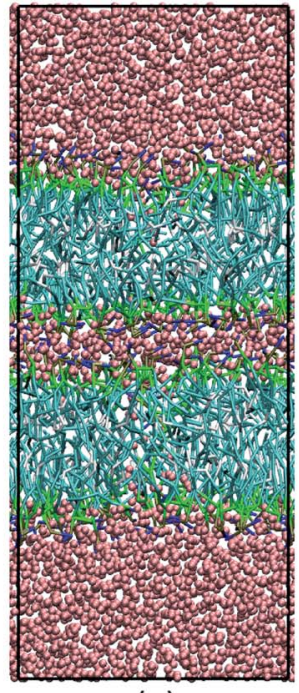

(c)

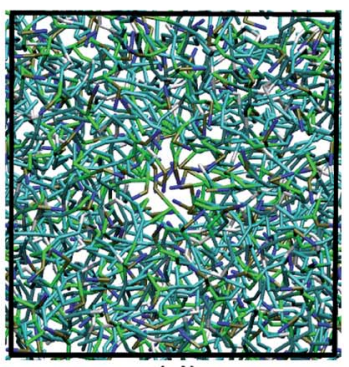

(d)

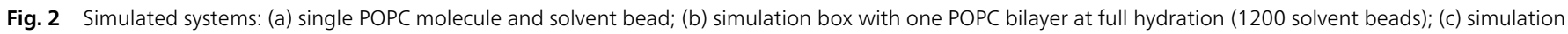
box for the umbrella sampling setup; (d) pore for the umbrella sampling setup. 
different numbers of water molecules per lipid, as shown in Table 1. In the first series all systems contained one bilayer with 128 lipids in the unit cell (small systems), and in the second series two opposed bilayers, each containing 512 lipids (large systems), were studied. The large systems were prepared by replication and parallel translation of the small systems in all three dimensions. Each small system was simulated for $1.2 \mu \mathrm{s}$ and each large system was simulated for $2.4 \mu \mathrm{s}$.

In order to calculate the hydration repulsion between bilayers mimicking grand canonical conditions the following setup was employed. The system at the initial configuration contained two bilayers (each comprising 128 lipids) separated by five water molecules per lipid, as indicated in Fig. 2c. A small pore in the middle of each bilayer (see Fig. 2d) was introduced such that water could diffuse through the bilayers to keep the chemical potential fixed for a set of distances between the bilayers. We stabilized these pores by applying cylindrical harmonic restraint acting only on the lipid tails. ${ }^{44} \mathrm{~A}$ water reservoir comprising about 105 water molecules per lipid was present to ensure a sufficient amount of solvent to cover the whole separation distance interval of interest and minimize the interaction between the bilayers across the periodic boundaries. The free energy landscape in terms of the potential of mean force (PMF) along a pre-chosen reaction coordinate was evaluated using the umbrella sampling method. ${ }^{45}$ The reaction coordinate employed was the distance between the centers of mass of the bilayers in the direction normal to the bilayer, $d_{\text {com }}$. The value of the reaction coordinate $d_{\text {com }}$ was restrained at 78 different equidistant values or umbrella "windows" in the interval from 4.7 to $7.4 \mathrm{~nm}$. In the initial configuration the number of solvent beads in the thin water layer between the opposing bilayers was 160. A weak umbrella potential with a force constant of $500 \mathrm{~kJ}$ $\mathrm{mol}^{-1} \mathrm{~nm}^{-2}$ was applied for $2.4 \mu \mathrm{s}$ in order to obtain the initial configurations for the umbrella windows. For the production runs a larger force constant of $5000 \mathrm{~kJ} \mathrm{~mol}^{-1} \mathrm{~nm}^{-2}$ was used in a series of $4.4 \mu \mathrm{s}$ simulations. To ensure sufficient relaxation of the reaction coordinate and the bilayer structure, only the final

Table 1 Simulated systems. The number of water molecules per lipid, $N_{w} / N_{\text {lip }}$ (note that the number of water molecules is equal to the number of solvent beads times four), and the area, $A$, for the small and large systems are given

\begin{tabular}{lll}
\hline$N_{\mathrm{w}} / N_{\text {lip }}$ & $A(\mathrm{small}) / \mathrm{nm}^{2}$ & $A($ large $) / \mathrm{nm}^{2}$ \\
\hline 37.5 & $41.08 \pm 0.03$ & $163.78 \pm 0.03$ \\
28.1 & $41.08 \pm 0.03$ & $163.78 \pm 0.04$ \\
25.0 & $41.08 \pm 0.05$ & $163.69 \pm 0.04$ \\
21.9 & $41.04 \pm 0.03$ & $163.55 \pm 0.05$ \\
20.3 & $40.94 \pm 0.03$ & $163.81 \pm 0.08$ \\
18.8 & $40.92 \pm 0.03$ & $162.93 \pm 0.03$ \\
17.2 & $40.68 \pm 0.02$ & $162.56 \pm 0.04$ \\
15.6 & $40.60 \pm 0.03$ & $161.81 \pm 0.04$ \\
14.1 & $40.33 \pm 0.05$ & $160.95 \pm 0.03$ \\
12.5 & $40.09 \pm 0.05$ & $159.99 \pm 0.03$ \\
10.9 & $39.77 \pm 0.02$ & $158.61 \pm 0.03$ \\
9.4 & $39.59 \pm 0.03$ & $157.32 \pm 0.03$ \\
7.8 & $38.62 \pm 0.02$ & $154.30 \pm 0.02$ \\
6.3 & $37.81 \pm 0.03$ & $152.17 \pm 0.09$ \\
5.0 & $37.48 \pm 0.03$ & $149.74 \pm 0.03$
\end{tabular}

$3.6 \mu$ s of the trajectories were used for analysis. The PMF profile was calculated from the distribution of the reaction coordinate in the biased ensemble using the weighted histogram analysis method. ${ }^{46}$

The bilayer isothermal area compressibility modulus, $K_{A}$, was calculated according to the following scheme. One fully hydrated initially tension-free bilayer, consisting of 128 lipids, was simulated at a membrane tension of $0.5,1-5$ (with the step of $1 \mathrm{mN} \mathrm{m}^{-1}$ ), 8, 10,12,15, and $20 \mathrm{mN} \mathrm{m}^{-1}$ and a normal pressure of 1 bar for $0.8 \mu \mathrm{s}$. From the final $400 \mathrm{~ns}$ of the trajectory the bilayer area, $A$, was calculated. For the area compressibility calculation the bilayer needed to be stretched such that the area change $\Delta A / A_{0}$ was about $8 \%$, which corresponds to the area change during dehydration in our simulations. Using a Hookean stress-strain relationship with the corresponding linear modulus $K_{A}$ yields

$$
\Sigma=K_{A} \frac{\Delta A}{A_{0}},
$$

where $\Sigma$ is the membrane tension. The latter can be calculated in the constant area ensemble from the average pressure components according to

$$
\Sigma=\left\langle L_{z}\left[P_{z z}-\frac{1}{2}\left(P_{x x}+P_{y y}\right)\right]\right\rangle,
$$

here \langle\rangle denotes an ensemble average and $L_{z}$ or $P_{z z} \equiv P_{N}$ are the box length or component of the pressure tensor normal to the membrane, respectively. $P_{x x}$ and $P_{y y}$ are the tangential components of the pressure tensor in the box and the lateral pressure is $P_{L}=\left(P_{x x}+P_{y y}\right) / 2$.

To estimate whether the water compressibility changes as the number of solvent beads/molecules in the system is reduced, additional simulations over a range of pressure values $(10,50,100,200$ and 300 bar) were performed to estimate the isothermal water compressibility, $\kappa$, using

$$
\kappa=\left.\frac{1}{\rho_{0}} \frac{\partial \rho}{\partial P}\right|_{T},
$$

where $\rho_{0}$ is the number density of the bulk water at $300 \mathrm{~K}$ and 1 bar. These simulations were conducted for $40 \mathrm{~ns}$ and for small systems with one bilayer and hydration corresponding to 37.5 , 12.5, 10.9, 9.4, 7.8, 6.3 and 5.0 water molecules per lipid.

Finally, to make a closer connection between coarse-grained water and atomistic water, simulations with the polarizable MARTINI water model ${ }^{47}$ were conducted. This model has the same level of coarse-graining, i.e. four water molecules mapped onto one coarse-grained particle but each particle has a three-bead representation. Two beads have equal charges of opposite sign and the central bead is neutral. Polarization effects are important at the bilayer/water interface. The polarizable water model allows us to study processes where electrostatic screening effects are important, such as permeation of ions across a membrane. The simulations were performed in the same ensemble and under the same conditions as for the small systems simulated with nonpolarizable water. The relative dielectric constant and effective time step were $\varepsilon=2.5$ and $120 \mathrm{fs}$, respectively. 


\section{Thermodynamic analysis of the repulsion pressure}

Repulsion pressure from changes in the area per lipid. First a single bilayer under no external potentials is considered. This simulation setup mimics one repeat unit of a periodic stack of bilayers with a fixed number of lipids and water molecules. At a given hydration level (the number of water molecules per lipid) and pressure $\left(P_{N}=P_{L}=1 \mathrm{bar}\right)$ the free energy of the system is minimized by properly adjusting the area of the box, $A$, and the box length, $L_{z}$. The repulsion forces tend to increase the interbilayer distance (i.e., the water layer thickness). As the lateral areas of the water and the bilayer are the same, this will lead to a decrease in the area per lipid. The balance will be limited by the finite area compressibility modulus of the bilayer, $K_{A}$. This effect can be described quantitatively as explained in the following. The free energy of the system in the $N A L_{z} T\left(V=A L_{z}\right)$ ensemble can be written as

$$
\Delta F=\Delta F_{\text {lip }}+\Delta F_{\mathrm{w}}+g\left(d_{\mathrm{w}}\right) A
$$

Here $\Delta F$ is the excess free energy with respect to a single isolated tensionless bilayer of $N_{\text {lip }}$ lipids embedded in bulk water. The symbol $\Delta F_{\text {lip }}$ denotes the free energy contribution due to the bilayer stretching or compression, $\Delta F_{\mathrm{w}}$ is the contribution due to the bulk water expansion or compression, and $g\left(d_{\mathrm{w}}\right)$ is the interface potential, i.e. the part of the free energy per unit area that is responsible for the interbilayer interactions which depend on the water space between bilayers, $d_{\mathrm{w}}$, with $\lim _{d \mathrm{w} \rightarrow \infty} g\left(d_{\mathrm{w}}\right)=0$. The free-energy change of the bilayer can be written using the area compressibility modulus, $K_{A}$, eqn (1), as

$$
\Delta F_{\text {lip }}=\frac{K_{A}}{2} \frac{\left(A-A_{0}\right)^{2}}{A_{0}},
$$

which implies that the excess free energy of the bilayer at zero surface tension is set to zero. By using the thermodynamic equation for the isothermal water compressibility, eqn (3), one arrives to similar equation for the free energy change for water due to its volume change

$$
\Delta F_{\mathrm{w}}=\frac{1}{2 \kappa} \frac{\left(V^{\mathrm{w}}-V_{0}^{\mathrm{w}}\right)^{2}}{V_{0}^{\mathrm{w}}},
$$

where $V_{0}^{W}$ is the volume of water corresponding to the bulk density at $T=300 \mathrm{~K}$ and pressure of 1 bar. However, the compressibility of water does not alter the final result, as shown in the Appendix. The functions of interest are $g\left(d_{\mathrm{w}}\right)$ and its first derivative, which are the interbilayer potential and the disjoining pressure between opposing membranes, respectively. In order to express the Helmholz free energy, eqn (4), in terms of its natural variables (the number of lipids $N_{\text {lip }}$, the number of water molecules $N_{\mathrm{w}}$, the area of the system $A$, the box size in the $Z$ direction $L_{z}$ and the temperature $T$ ) the water layer thickness is written as

$$
d_{\mathrm{w}}=L_{z}-d_{\mathrm{hh}},
$$

where $d_{\mathrm{hh}}$ denotes the bilayer thickness for which

$$
d_{\mathrm{hh}}=\frac{N_{\mathrm{lip}}}{\rho_{\mathrm{hh}} A}
$$

holds and we assume that the number density of the hydrophobic part of the bilayer $\rho_{\mathrm{hh}}$ does not depend on the number of water molecules (this assumption will be verified via simulations, see Results section). Combining eqn (7) and (8) yields

$$
V^{\mathrm{w}}=d_{\mathrm{w}} A=L_{z} A-\frac{N_{\text {lip }}}{\rho_{\mathrm{hh}}}
$$

for the water volume. Finally, for the excess free energy of the system the expression

$$
\Delta F=\frac{K_{A}}{2} \frac{\left(A-A_{0}\right)^{2}}{A_{0}}+g\left(L_{z}-\frac{N_{\mathrm{lip}}}{\rho_{\mathrm{hh}} A}\right) A+\frac{\left(L_{z} A-N_{\mathrm{lip}} / \rho_{\mathrm{hh}}-V_{0}^{\mathrm{w}}\right)^{2}}{2 \kappa V_{0}^{\mathrm{w}}}
$$

is obtained. As our simulations are performed in the NTP ensemble, we apply a Legendre transformation of eqn (10) to obtain an expression for the excess Gibbs free energy

$$
\begin{aligned}
G\left(N_{\text {lip }}, N_{\mathrm{w}}, P_{N}, P_{L}, T\right) & \equiv F-\left.A \frac{\partial F}{\partial A}\right|_{L_{z}}-\left.L_{z} \frac{\partial F}{\partial L_{z}}\right|_{A} \\
& =F+\left(P_{L}+P_{N}\right) V, \\
\Delta G\left(N_{\text {lip }}, N_{\mathrm{w}}, P_{N}, P_{L}, T\right) & =\Delta F+\left(P_{L}+P_{N}\right) \Delta V .
\end{aligned}
$$

where $\Delta V$ is the volume change between a single bilayer comprised of $N_{\text {lip }}$ lipids and $N_{\mathrm{w}}$ water molecules under periodic boundary conditions (i.e., one repeat unit of a stack of bilayers) and an isolated bilayer of $N_{\text {lip }}$ lipids and $N_{\mathrm{w}}$ bulk water molecules (in contact with bulk water) at the specified pressures. In our simulation setups for all hydration levels the condition $P_{N}=$ $P_{L}=1$ bar is satisfied, and as for the dense fluids the term $P \Delta V$ $\approx 0$ can be neglected. Therefore we have $\Delta G=\Delta F$. In order to determine the disjoining pressure at a given hydration level (or at given $d_{\mathrm{w}}$ ), the excess free energy has to be minimized with respect to $d_{\mathrm{w}}$, as shown in the Appendix. The repulsion pressure $P\left(d_{\mathrm{w}}\right) \equiv-g^{\prime}\left(d_{\mathrm{w}}\right)$ can then be obtained from

$$
P\left(d_{\mathrm{w}}\right)=\frac{K_{A}}{d_{\mathrm{w}}}\left(1-\frac{A}{A_{0}}\right) .
$$

This equation for the disjoining pressure is valid under the condition of equal lateral and normal pressures, and the area and the water layer thickness are ensemble averaged values. A similar equation was obtained in ref. 25 under the additional assumption that water is incompressible.

The experiment corresponds to an ensemble where $N_{\text {lip }}, \mu_{\mathrm{w}}$, $P_{N}$, and $P_{L}$ are fixed, and the area, $A$, and the periodicity, $L_{z}$, of the membrane stack adjust in turn. As the bilayer stack can freely exchange water molecules with the vapor phase, the vapor phase and the bilayer stack are characterized by the same chemical potential, $\mu_{\mathrm{w}}$. The thermodynamic relationship, $N_{\mathrm{w}}=\left.V \frac{\partial P_{\mathrm{w}}}{\partial \mu_{\mathrm{w}}}\right|_{V T}$, provides a relationship between the osmotic pressure and the chemical potential of the water vapor, $\Delta P_{\mathrm{w}} \approx \frac{1}{v_{\mathrm{w}}} \Delta \mu_{\mathrm{w}}$, where $v_{\mathrm{w}}$ is the partial volume of water. One can show that this osmotic pressure equals the disjoining pressure. ${ }^{41}$ Here we do not exploit the relationship between disjoining and osmotic pressure but invoke the equivalence of 
different ensembles in the thermodynamic limit to apply the relationship, eqn (13), between the experimentally observed changes in the bilayer area and thickness in order to evaluate the interbilayer repulsion inducing these structural changes.

Controlling the center-of-mass distance between the membranes. As an alternative approach to validate the disjoining pressure, we consider two bilayers with an umbrella potential on the distance between the centers of mass of the bilayers, $U\left(d_{\text {com }}\right)=k\left(d_{\text {com }}-d_{\text {com }, 0}\right)^{2} / 2$. This approach may mimic (i) SFA measurements as well as (ii) the SNARE mediated approach of two membranes during the initial stages of membrane fusion. Differences would arise, though, first, from the fact that our umbrella sampling simulations are applied to two free-standing bilayers, whereas in SFA experiments the membranes are immobilized on a solid support. This means that lipid protrusions are suppressed in SFA experiments but not in our umbrella sampling simulations. Second, whereas in our simulations an external potential is applied to the centers of mass of the bilayers as a global restraint, SNARE proteins would apply forces locally at their anchoring points. Local force application may distort the bilayers and facilitate bilayer fusion. In fact it has been proposed that the local perturbation of the membrane close to the transmembrane anchors of the SNARE proteins for close intermembrane distances may be one of the mechanisms by which SNARE proteins induce membrane fusion. ${ }^{48,49}$ If the umbrella potential is applied to the center-ofmass distance between the bilayers, $d_{\text {com }}$, the excess free energy of the double bilayer system is

$$
\Delta F\left(A, d_{\mathrm{com}}\right)=K_{A} \frac{\left(A-A_{0}\right)^{2}}{A_{0}}+F_{\mathrm{h}}\left(d_{\mathrm{w}}\right)+U\left(d_{\mathrm{com}}\right),
$$

where the water compressibility has been neglected and $F_{\mathrm{h}}\left(d_{\mathrm{w}}\right)$ denotes the contribution from the interbilayer repulsion to the free energy. As we are interested in the excess free energy the contribution due to the pore potential is neglected as well.

The symbol $f\left(d_{\mathrm{w}}\right)=F_{\mathrm{h}}\left(d_{\mathrm{w}}\right) / A$ shall denote the repulsion free energy per unit area at a given water layer thickness $d_{\mathrm{w}}$. A Legendre transformation and the arguments used in the last section lead to $f\left(d_{\mathrm{w}}\right)=g\left(d_{\mathrm{w}}\right)$, where $g\left(d_{\mathrm{w}}\right)$ denotes the interface potential at constant pressure (1 bar) lateral and normal to the membranes and is identical to the function $g\left(d_{\mathrm{w}}\right)$ given in eqn (4). The distance between the centers of the mass of the two bilayers is $d_{\mathrm{com}}=d_{\mathrm{w}}+d_{\mathrm{hh}}$. The bilayer thickness and the area of the bilayer are related $v i a d_{\mathrm{hh}}=V_{\mathrm{bil}} / A$, where $V_{\mathrm{bil}}$ is the volume of one bilayer. At equilibrium, $\partial \Delta F / \partial A=\partial \Delta F / \partial d_{\mathrm{com}}=0$. We consider that $\partial d_{\mathrm{w}} / \partial A=V_{\mathrm{bil}} / A^{2}$ and $P\left(d_{\mathrm{w}}\right)=-\left(\partial F_{\mathrm{h}} / \partial d_{\mathrm{w}}\right) / A_{\text {eq }}$ with $P\left(d_{\mathrm{w}}\right)$ given by eqn (13) replacing $A$ by $A_{\mathrm{eq}}$ (renaming the variable without changing its meaning). Furthermore, we note that the negative gradient of the PMF $\left(d_{\text {com }}\right)$ and the umbrella potential force balance each other on average, that is

$$
\operatorname{PMF}^{\prime}\left(d_{\text {com }, 1}\right)+k\left(d_{\text {com }}-d_{\text {com }, 0}\right)=0 .
$$

Here, $d_{\text {com }}$ denotes the average center-of-mass distance observed for the given umbrella window and $d_{\mathrm{com}, 1}$ is some value between $d_{\mathrm{com}, 0}$ and $d_{\mathrm{com}}$. In the asymptotic limit of large $k$, $d_{\mathrm{com}, 1} \approx d_{\mathrm{com}} \approx d_{\mathrm{com}, 0}$. In this limit, the equations

$$
\begin{gathered}
A_{\text {eq }}\left(d_{\mathrm{w}}\right)=A_{0}-\frac{2 d_{\mathrm{w}}}{d_{\mathrm{com}}-d_{\mathrm{w}}}\left(A\left(d_{\mathrm{com}}\right)-A_{0}\right), \\
P\left(d_{\mathrm{w}}\right)=-\frac{1}{A_{\text {eq }}\left(d_{\mathrm{w}}\right)} \operatorname{PMF}^{\prime}\left(d_{\mathrm{com}}\right)
\end{gathered}
$$

are obtained. Here, $P\left(d_{\mathrm{w}}\right)=-g^{\prime}\left(d_{\mathrm{w}}\right)$ and $A_{\mathrm{eq}}=A_{\text {eq }}\left(d_{\mathrm{w}}\right)$ denotes the membrane area at a distance between the membrane surfaces $d_{\mathrm{w}}$ when the water content is controlled. This equation system relates the area of the membrane patch $A_{\text {eq }}$ and the potential of mean force profile PMF $\left(d_{\mathrm{com}}\right)$ at given $d_{\mathrm{w}}$ both obtained from the umbrella sampling simulations to the repulsion pressure $P\left(d_{\mathrm{w}}\right)$ inferred from the changes in the area per lipid at equilibrium.

These equations have two interesting implications. The first equation shows that with decreasing $d_{\mathrm{w}}$ the area of the membrane patch in the umbrella sampling, $A$, and in the equilibrium simulations, $A_{\mathrm{eq}}$, change in opposite ways; whereas decreasing $d_{\mathrm{w}}$ leads to a decrease in $A_{\mathrm{eq}}$ as shown in the previous section, reduction in $d_{\mathrm{w}}$ results in an increase in $A$. The latter effect can be understood qualitatively from the following considerations. For a given position of the umbrella potential, $d_{\text {com }, 0}$, the contribution from the interbilayer repulsion, $P\left(d_{\mathrm{w}}\right)$, to the free energy of the system, $\Delta F\left(A, d_{\text {com }}\right)$, is decreased by an increase in the distance between the membrane surfaces, $d_{\mathrm{w}}$. As $d_{\mathrm{w}}=d_{\mathrm{com}}-d_{\mathrm{hh}}$ and $d_{\mathrm{com}} \approx d_{\mathrm{com}, 0}$ for the large force constant $k$ of the umbrella potential, an increase in $d_{\mathrm{w}}$ is mainly possible due to a decrease in $d_{\mathrm{hh}}$, i.e., thinning of the membrane. As the lipids are largely incompressible, this is accompanied by an increase in the membrane area, $A$. This effect is balanced by the finite area compressibility modulus $K_{A}$ of the bilayers.

The second equation shows that if properly normalized in terms of membrane area $\left(A_{\text {eq }}\right.$ versus $\left.A\right)$, corresponding reaction coordinates are properly related to each other $\left(d_{\mathrm{com}}=d_{\mathrm{w}}+V_{\mathrm{bil}} / A\right)$, and if $k$ is chosen sufficiently large, both approaches should yield identical results. In practice, the choice of $k$ is a tradeoff between the large $k$ limit needed for accuracy and the need to keep $k$ reasonably small such that the number of umbrella windows needed to ensure overlap between neighboring windows is not too large. The equilibrium method, on the other hand, does not suffer from this required tradeoff and is accurate for an arbitrary choice of intermediate hydration levels chosen.

\section{Results and discussion}

\section{Area compressibility modulus}

In order to calculate the disjoining pressure between membranes using eqn (13) the area compressibility modulus for the coarse-grained model must be determined. The area compressibility modulus (or zero-tension stretching modulus, or membrane compression modulus) was calculated in several experimental studies ${ }^{50,51}$ and using computer simulations for atomistic (ref. 52 and references therein) as well as coarsegrained lipid models. ${ }^{53,54}$ Experimental values for phospholipid bilayers are in the range of $180-330 \mathrm{mN} \mathrm{m}^{-1}$, while coarsegrained values are somewhat smaller $\left(70-140 \mathrm{mN} \mathrm{m}^{-1}\right)$ and atomistic values are somewhat larger $\left(404 \mathrm{mN} \mathrm{m}^{-1}\right)$. 


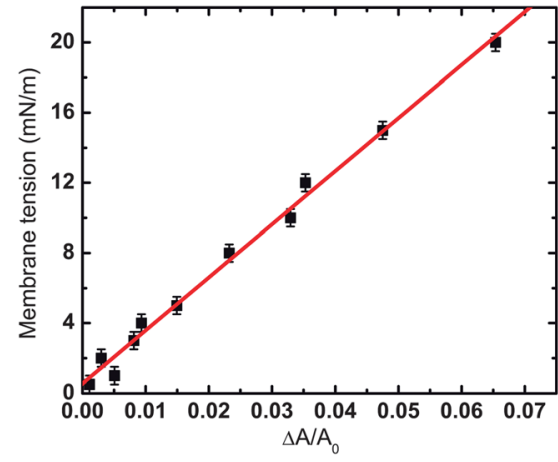

Fig. 3 Membrane tension $\Sigma$ versus relative area change $\Delta A / A_{0}$. Symbols represent simulation data and the line is a linear fit.

Near the free-energy minimum the change $\Delta A$ in the equilibrium membrane area as a function of membrane tension $\Sigma$ can be related to the area compressibility modulus $K_{A}$ according to eqn (1). Fig. 3 shows the membrane tension versus the relative area change at full hydration. Fitting the data in Fig. 3 to eqn (1) yields $K_{A}=303 \pm 9 \mathrm{mN} \mathrm{m}^{-1}$, which is within the experimental range. The area compressibility modulus can also be calculated from the thermal area fluctuations. As system box sizes used in simulations are typically not large, contribution due to membrane undulations can be neglected and these two methods should give similar results. Indeed, the area compressibility modulus for a POPC bilayer was also calculated from the area fluctuations using the same coarse-grained model but yielding a higher value of $539 \pm 33 \mathrm{mN} \mathrm{m}^{-1}{ }^{55}$ Here it must be noted that the fluctuation approach is expected to be less precise due to insufficient sampling ${ }^{51,53}$ and care has to be taken to use a proper thermodynamic ensemble. Although the Berendsen weak coupling method used to control the temperature and pressure in ref. 55 yields correct average properties, it is not able to reproduce the fluctuations of volume/area in the NPT ensemble. Therefore, the fluctuation approach should be used in conjunction with the Nose-Hoover thermostat ${ }^{56,57}$ and the Parinello-Rahman barostat. ${ }^{58}$

\section{The area per lipid, bilayer interface and thickness}

Fig. 4 shows the changes in the area per lipid with dehydration in the unrestrained simulations for both system sizes, for atomistic simulations, ${ }^{26}$ and our simulations with the umbrella potential. For the umbrella sampling simulations, the area of the bilayer was calculated from the actual area of the box minus the pore area and is estimated to be $2 \mathrm{~nm}^{2}$. (Additionally, a simulation of the system at full hydration, i.e. large distance between the bilayers, and without an external pore potential was conducted. The pore area was estimated as the difference between the areas with and without an external pore potential.) In these simulations, the area per lipid and the bilayer thickness showed large fluctuations. The dotted line represents the experimental area per lipid at full hydration for a POPC bilayer from ref. 59. In our experiments the area per lipid at different hydration levels is not straightforward to obtain. We notice that the equilibrium area per lipid of

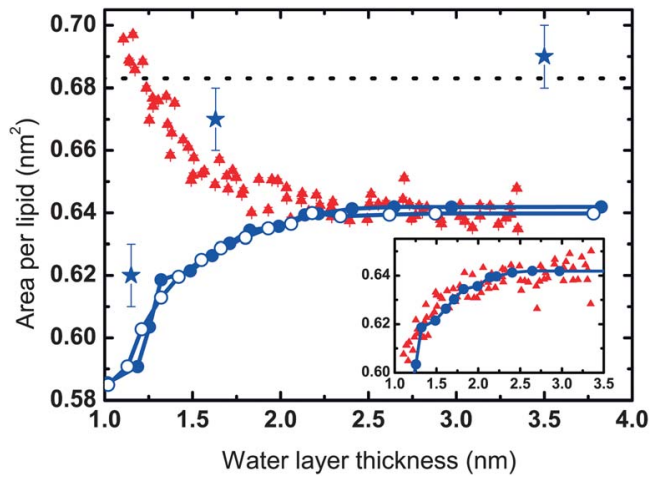

Fig. 4 Area per lipid versus water layer thickness for coarse-grained simulations of small (solid circles) and large systems (open circles), atomistic ${ }^{26}$ (stars) as well as umbrella sampling simulations (solid triangles) and the experimental value (dotted line) at full hydration. ${ }^{59}$ The error bars for the equilibrium data points are smaller than the size of the circles. The area per lipid changes upon dehydration for the small system (solid circles) using eqn (16) (solid triangles) are shown in the inset.

$0.693 \mathrm{~nm}^{2}$ at high hydration levels from atomistic simulations is closer to the experimental value of $0.683 \mathrm{~nm}^{2}$ than to our full hydration value of $0.64 \mathrm{~nm}^{2}$. The discrepancy in the area per lipid between the CG and the atomistic simulations decreases with decreasing hydration level.

Strikingly, a decrease in the hydration level without restraint leads to a decrease in the area per lipid in our CG as well as in atomistic simulations. In contrast, when the umbrella potential is applied to the center-of-mass distance between the bilayers, dehydration leads to an increase in the area per lipid, in agreement with eqn (16). The area per lipid change for the unrestrained setup was calculated using the area per lipid change measured from umbrella sampling simulations, the results are shown in the inset of Fig. 4.

Considering that lipid bilayers are nearly incompressible in terms of volume, changes in the area per lipid with dehydration are associated with opposite changes in the membrane thickness. Validation of the membrane thickness requires choosing a definition for the position of the interface between the bilayer and the water phase. Several ways to define a bilayer thickness have been reported in the literature. In an atomistic simulation study of POPC bilayers ${ }^{26}$ the interface was defined by the Gibbs dividing surface between the water and the lipids. In X-ray studies the thickness is typically defined as the distance between the maxima in the electron densities. ${ }^{21,59}$ These maxima are often related to the electron-dense phosphate peaks. Here we will use the latter definition of the lipid/water interface, since we want to compare our simulation results with the experiments. In Fig. 5 a the partial mass densities of water, lipids, phosphates and glycerol backbones are plotted for the bilayer at full hydration. Each lipid/water interface could be defined as the position of the corresponding maximum of the phosphate density. However, here we define the interface at equal densities of phosphate and glycerol. With this definition the bilayer thicknesses are closer to the corresponding experimental values. Fig. 5b shows the change in the bilayer thickness upon dehydration for the two alternative definitions of the interface. The water layer thickness for the small system size is defined as the box size in the $Z$ direction minus the bilayer 

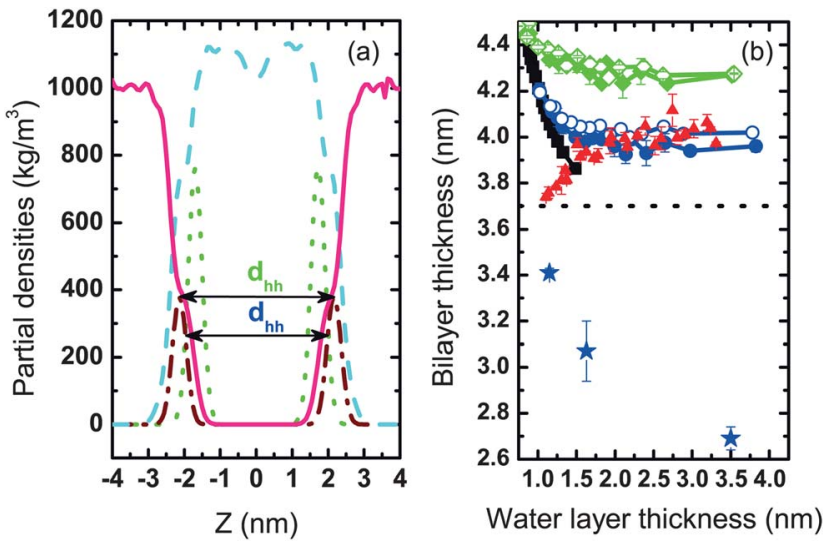

Fig. 5 (a) Partial mass densities of water (full line), phosphate (dash-dotted line), glycerol (dotted line), and lipids (dashed line) normal to the bilayer averaged over 800 ns for a single bilayer at full hydration. Two alternative definitions for the bilayer thickness are indicated with arrows. (b) Bilayer thickness versus water layer thickness for the experiment (solid squares), coarse-grained simulations without restraint using small (solid circles and rhombi) and large (open circles and rhombi) systems, as well as for the umbrella sampling simulations (solid triangles) and atomistic simulations from ref. 26 (stars). The dotted line represents the POPC bilayer thickness at full hydration from ref. 59. Rhombi are for the thickness defined from the maxima positions in the phosphate density and circles are for the phosphate glycerol equal density positions.

thickness. As the experimental measurements performed in the present work were only for low hydration levels, the POPC bilayer thickness at full hydration (dotted line) was taken from ref. 59. The system size effect on the bilayer thickness is also shown. For the small system at full hydration the bilayer thickness is $3.95 \pm$ $0.03 \mathrm{~nm}$ and for the large system it is $4.02 \pm 0.01 \mathrm{~nm}$ which is close to the experimental value of $3.7 \mathrm{~nm}$. The effective membrane "thickening" at large system size can be rationalized by larger undulations (see the ESI for the additional information on the membrane thickness change $\dagger$ ). Atomistic simulation results for the bilayer thickness from ref. 26 are shown with stars. Note that in ref. 26 only the mass densities of water and lipids were plotted. However, the positions of the maxima of the lipid electron densities are equivalent to the positions of the maximal lipid mass densities calculated from atomistic simulations.

\section{Disjoining pressure profiles}

Fig. 6 shows semilogarithmic plots of disjoining pressures versus water layer thickness, $d_{\mathrm{w}}$, using data from our experiments and simulations, atomistic simulations from ref. 26 , as well as experimental results from ref. 6 . The simulation data for the small and the large system are very similar and the difference between them is an estimate of systematic errors. The repulsion pressure profile obtained from our umbrella sampling simulations is shown as well. Solid triangles indicate results calculated using eqn (17).

Experimental and simulation data are in good agreement. A linear fit to the simulation data in the interval $d_{\mathrm{w}}=1.3-1.9 \mathrm{~nm}$ leads to a decay length of $\lambda_{t}=0.28 \pm 0.03 \mathrm{~nm}$ for the small and $0.30 \pm 0.01 \mathrm{~nm}$ for the large system without restraint, and 0.28 $\pm 0.01 \mathrm{~nm}$ for the umbrella sampling setup. Hence, the decay lengths observed in our simulations under different boundary

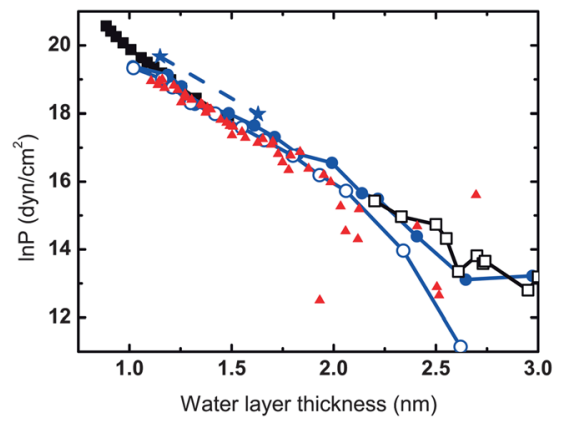

Fig. 6 Disjoining pressure versus water layer thickness in semilogarithmic presentation. Note that $1 \mathrm{dyn} \mathrm{cm}^{-2}=10^{2} \mathrm{mN} \mathrm{m}^{-2}$. Solid squares indicate our experimental results and open squares indicate findings adopted from ref. 6 . Solid circles represent data from our small system simulations and open circles represent those from the large system simulations both without restraint. Solid stars represent points obtained from atomistic simulations ${ }^{26}$ using eqn (13) and solid triangles represent data from umbrella sampling simulations using eqn (17). The difference between the data points for small and large systems is an estimate of systematic errors.

conditions are the same within the statistical error. The value for the decay length from our experimental data is $0.22 \pm 0.01$ $\mathrm{nm}$, which is close to the value of $0.26 \pm 0.01 \mathrm{~nm}$ from ref. 50 .

The decay of the disjoining pressure is the superposition of the decay of the hydration and the protrusion component which will typically have different values. The decay of the hydration component might be related to the correlation length $\xi$ of density fluctuations of water in the model employed here. The value of $\xi$ for the coarse-grained MARTINI water was estimated using an Ornstein-Zernike fit to the collective structure factor of bulk water $I(q)=\frac{I(0)}{1+q^{2} \xi^{2}}$, where $q$ is the scattering vector and $I(0)$ denotes the scattering intensity at zero with $I(0)=\frac{\left\langle(\Delta N)^{2}\right\rangle}{\langle N\rangle}=k_{\mathrm{B}} T \rho_{0} \kappa$, yielding $\xi=0.15 \mathrm{~nm}$. Thus the nearly single-exponential decay of repulsions is characterized by a length of the same order of magnitude as the bulk correlation length of the MARTINI water. For comparison, the correlation length for atomistic SPC/E water at $298 \mathrm{~K}$ is about $0.21 \mathrm{~nm}$ (from the Ornstein-Zernike fit of the scattering function using results from ref. 60). The smaller size of the bulk correlation length compared to the atomistic SPC/E water presumably arises from the coarse-grained nature of the model.

Disjoining pressure calculations using eqn (13) and data from atomistic simulations ${ }^{\mathbf{2 6}}$ are shown with stars. For the area compressibility modulus the value of $400 \mathrm{mN} \mathrm{m}^{-1}$ from atomistic simulations of the POPC bilayer at full hydration with the Berendsen thermostat ${ }^{52}$ is employed. Due to lack of simulation data only two points are available, connecting these points by a line gives an estimate for the decay length of $0.28 \mathrm{~nm}$. The overestimation of the amplitude of the interbilayer repulsion might be due to the overestimation of the area compressibility modulus $K_{A}$ from the area fluctuations in simulations using the Berendsen barostat. As pointed out above, such a procedure as applied in ref. 55 will overestimate $K_{A}$ by some factor $s$. A more accurate approach is to plot the membrane tension over the membrane area as done in the present paper. A comparison of 
our result with the one from ref. 55 suggests $s=0.56$. Scaling $K_{A}$ from Poger's simulations by $s$ gives $K_{A}=224$. Interestingly, the interbilayer repulsion deduced using this value overlaps exactly with our results.

At large distances between the membranes the disjoining pressure is small such that the signal-to-noise ratio becomes very low. This is the reason why the simulation data scatter at larger distances, $d_{\mathrm{w}}>2 \mathrm{~nm}$, on the logarithmic scale. In experiments, at these distances, other components of intermembrane forces start to play a role, such as entropic repulsion due to membrane undulations and peristaltic deformations, and van-der-Waals attraction. In simulations long range forces are typically underestimated due to the cutoffs used in the force fields and small bilayer patches.

\section{Water compressibility near the interface}

At low hydration levels with $d_{\mathrm{w}}<1.3 \mathrm{~nm}$ there is no bulk water in the system, so that all water molecules are bound to the head groups and the head groups from different bilayers are significantly overlapping. This water has different properties than bulk water, such as a decreased diffusion constant and increased compressibility. In ref. 61 it was shown that water near large hydrophobic solutes shows larger fluctuations with a lower density and a higher compressibility than bulk water. This effect increases with increasing solute size and hydrophobicity. Fig. 7 shows how the density of water near the bilayer surface changes with dehydration. The density of lipids remains almost constant, the value at the hydrophobic core changes by only $1.8 \%$ upon dehydration, whereas the density in the head group region (at $2 \mathrm{~nm}$ from the bilayer center) changes by $23.5 \%$. The product $d_{\mathrm{hh}} A$ at low hydration $\left(d_{\mathrm{w}}=1 \mathrm{~nm}\right)$ decreases by only $2 \%$ compared to that at full hydration indicating that the hydrophobic core and water remain nearly incompressible. The nevertheless finite water compressibility was calculated using eqn (3) where for each hydration level $\rho_{0}$ was taken as the maximal

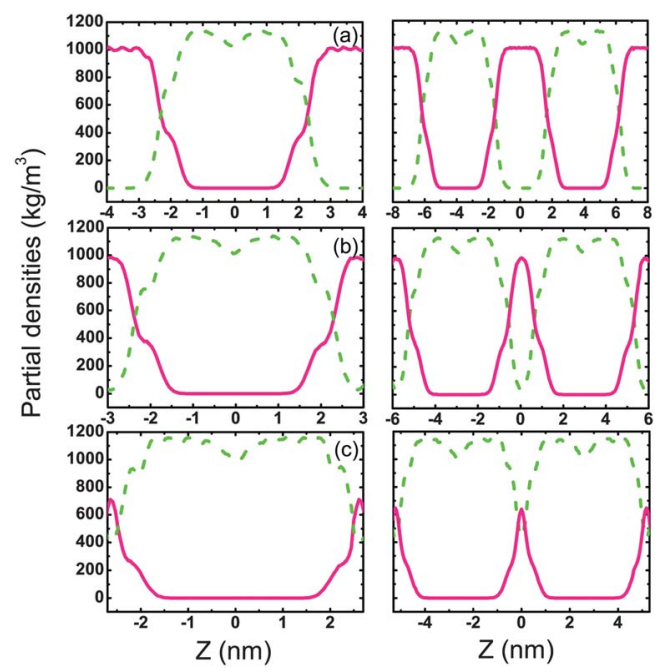

Fig. 7 Partial mass densities of water (full line) and lipids (dashed line) normal to the bilayer averaged over 800 ns for the single (left column) and the two bilayer (right column) system at (a) 37.5, (b) 15.6, and (c) 5 water molecules per lipid. The mass of the CG beads is $72 \mathrm{u}$ independent of the bead type.
Table 2 Water compressibility at different hydration levels

\begin{tabular}{lc}
\hline$N_{\mathrm{w}} / N_{\text {lip }}$ & $\kappa \times 10^{-5} \mathrm{bar}^{-1}$ \\
\hline 37.5 & $8.9 \pm 0.1$ \\
12.5 & $8.5 \pm 0.1$ \\
10.9 & $5.3 \pm 0.1$ \\
9.4 & $13.3 \pm 0.1$ \\
7.8 & $16.9 \pm 0.2$ \\
6.3 & $24.2 \pm 0.3$ \\
5.0 & $43.5 \pm 0.6$ \\
\hline
\end{tabular}

value of the water density at $P=1$ bar and the results are compiled in Table 2. At full hydration the compressibility is about $8 \times 10^{-5}$ bar $^{-1}$ (which is slightly higher than the value for atomistic water, 4.6-5.5 $\times 10^{-5}$ bar $^{-1}$ (ref. 24 and 60)) and, at the hydration of 9.4 water molecules per lipid, it is $13 \times 10^{-5} \mathrm{bar}^{-1}$. The compressibility at hydration of 7.8 water molecules per lipid is twice as large as that at full hydration. For systems with even lower water content, the compressibility grows dramatically, see Table 2, meaning that all water molecules become bound to lipid heads and cannot be treated as a separate phase.

\section{Free-energy decomposition}

Fig. 8 shows the decomposition of the Gibbs free-energy profiles from the unrestrained simulations (large system size, Fig. 8a) and the umbrella sampling simulations (Fig. 8b) into their enthalpic, $H$, and entropic, $-T S$, contributions. The free energies and the contributions are normalized by the membrane area. The enthalpic contribution was estimated from the total potential energy of the system, as the contribution from the change in the volume is negligible. For low hydration levels, the total enthalpy for the large system was calculated as the system enthalpy plus $\Delta H_{\mathrm{W}}=\Delta N_{\mathrm{w}} h_{\mathrm{w}}$, where $\Delta N_{\mathrm{w}}$ is the number of water molecules removed from the fully hydrated system and $h_{\mathrm{w}}=$ $22.8 \pm 0.5 \mathrm{~kJ} \mathrm{~mol}^{-1}$ is the enthalpy per solvent bead in the bulk.

Our results shall be compared with the recent findings by Schneck et al. ${ }^{24}$ who applied an atomistic model and dehydrated a bilayer stack at a constant membrane area $A$. As dehydration leads to a decrease in $A$ without restraint but an increase in $A$ when the bilayers are pushed together, the conditions considered by Schneck et al. are intermediate to the extreme conditions employed in the present study. Schneck et al. decomposed their free energy profiles for the interbilayer repulsion into the direct contribution from the interaction of lipids (inter- and intramembrane interactions), $G_{\mathrm{dir}}$, and the water mediated contribution, $G_{\text {ind }}$, including water-water and water-lipid interactions. Both contributions were further subdivided into their respective enthalpic, $H_{\text {dir }}$ and $H_{\text {ind }}$, and entropic components, $-T S_{\mathrm{dir}}$ and $-T S_{\text {ind }}$.

As in the model employed in our study, the water is represented by isotropic beads, the entropic contribution, $-T S$, presumably arises mainly from the lipids, i.e., $-T S \approx-T S_{\text {lip. }}$ (It is assumed that not the same water molecules are bound to lipid headgroups all the time, but the water molecules will be dynamically exchanged, such that there will be no effect of these water molecules on the entropy.) The entropic contribution from the lipids contains components from the inter- and 

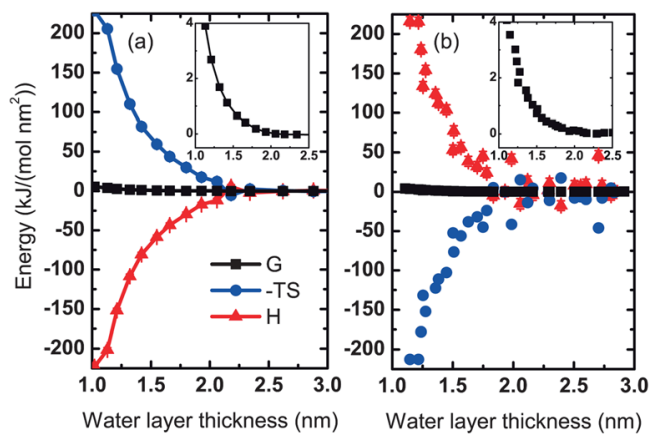

Fig. 8 Free energy decomposition for the (a) large system without restraint and (b) umbrella sampling system. The error bars are depicted for all data points, for some data points the error bars are smaller than the size of the symbols. The zoomed-in free energy profile is shown in the inset of each plot. Note that 1 dyn $\mathrm{cm}^{-1}=0.6 \mathrm{~kJ} \mathrm{~mol}^{-1} \mathrm{~nm}^{-2}$

intramembrane interactions, $-T S_{\text {ter }}$ and $-T S_{\text {tra }}$, respectively, according to $-T S_{\text {lip }}=-T S_{\text {ter }}-T S_{\text {tra. }}$ Likewise, the enthalpic components of lipid-lipid interactions, $H_{\text {lip }}$, contain contributions from inter- and intrabilayer interactions, $H_{\text {ter }}$ and $H_{\text {tra }}$, respectively, according to $H_{\text {lip }}=H_{\text {ter }}+H_{\text {tra }}$. The remaining enthalpic contributions arise from lipid-water, $H_{\text {lip-wat }}$, and water-water, $H_{\text {wat-wat }}$, interactions.

In their free-energy decomposition, Schneck et al. did neither present further decomposition of the indirect interaction into water-water and water-lipid interactions, nor further decomposition of $S_{\mathrm{dir}}$ into $S_{\mathrm{tra}}$ and $S_{\mathrm{ter}}$. This gap shall be filled by our work.

Although the free-energy profiles (normalized by the membrane area) from both approaches are very similar they arise from very different enthalpy-entropy balances as displayed in Fig. 8. Fig. 8a shows that dehydration without restraint leads to a decrease in $H$ and an increase in $-T S$ $\left(\approx-T S_{\text {lip }}\right.$, implying a decrease in $\left.S_{\text {lip }}\right)$. Fig. 9a reveals that the decrease in $H$ arises from a decrease in $H_{\text {lip }}$. The latter is partially due to a reduction in $H_{\text {ter }}$, as observed by Gentilcore et al. ${ }^{19}$ and Schneck et al. ${ }^{24}$ However, our analysis reveals also a decrease in $H_{\text {tra }}$ which is even larger than the change in $H_{\text {ter }}$. The decrease in $H_{\text {tra }}$ in our simulations presumably arises from the thickening of the membranes which leads to increased favorable dispersion interactions between the lipids.
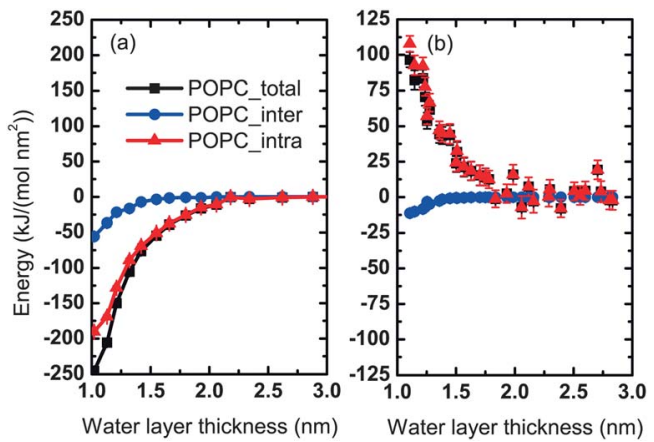

Fig. 9 Enthalpy decomposition for lipid interactions into inter- and intramembrane contributions as well as the sum of these components for the (a) large system without restraint and (b) umbrella sampling system. For some data points the error bars are smaller than the size of the symbols.

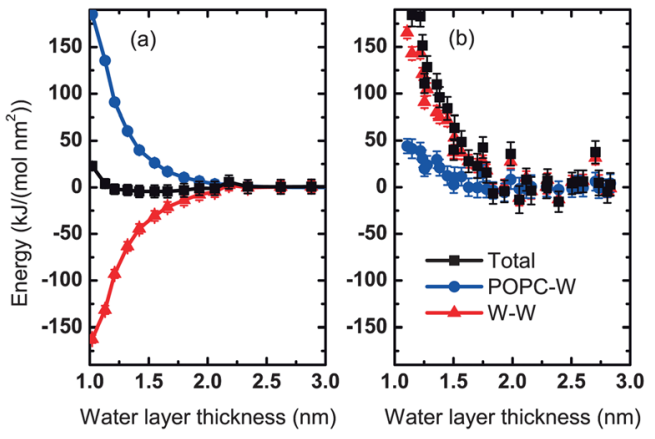

Fig. 10 Enthalpy decomposition for water interactions: water-lipid and waterwater interactions as well as the sum of these two contributions for the (a) large system without restraint and (b) umbrella sampling system. For some data points the error bars are smaller than the size of the symbols.

As can be seen in Fig. 10a, the decrease in $H_{\text {lip }}$ is partially compensated by an increase in $H_{\text {lip-wat }}+H_{\text {wat-wat }}$. The latter arises from a strong rise in $H_{\text {lip-wat }}$ which has also been observed in ref. 24. The latter is presumably due to the desorption of water from the lipids through the transfer of water from the intermembrane space to the bulk which, in contrast, is expected to increase the favorable water-water interactions. Indeed, as shown in Fig. 10a, the increase in $H_{\text {lip-wat }}$ is largely, albeit not fully, compensated by a decrease in $H_{\text {wat-wat }}$.

As $S_{\text {lip }}=S_{\text {ter }}+S_{\text {tra }}$, the decrease in $S_{\text {lip }}$ is due to a net decrease in the sum of $S_{\text {ter }}$ and $S_{\text {tra }}$. A decrease in $S_{\text {ter }}$ was also observed in ref. 24, where it was explained by a zippering process, which leads to a correlation of lipid headgroups of the proximate leaflets of opposing bilayers. Presumably, dehydration also leads to a decrease in $S_{\text {tra }}$ due to (i) the suppression of protrusion modes ${ }^{12}$ and (ii) the increase in membrane thickness which implies an increase in the order of the lipid tails.

Fig. 8b shows that for the umbrella sampling setup, strikingly, the entropy-enthalpy balance is reversed compared to the unrestrained simulations, i.e., dehydration leads to an increase in $H$ but a decrease in $-T S$ (implying an increase in $S=S_{\text {lip }}$ ). From Fig. $9 \mathrm{~b}$ it is seen that dehydration leads to a reduction in $H_{\text {ter }}$ like in the unrestrained simulations, although with smaller magnitude. Unlike without restraint, however, $H_{\text {tra }}$ grows with dehydration. This overcompensates the reduction in $H_{\text {ter }}$ such that $H_{\text {lip }}$ overall increases, which emphasizes the importance of considering $H_{\text {tra }}$ in the free-energy decomposition. The increase in $H_{\text {tra }}$ presumably stems from the decrease in the size of attractive dispersion interactions between lipid tails due to the stretching of the bilayer. $H_{\text {lip-wat }}$ again grows upon dehydration but to a much lower extent than in the unrestrained simulations, see Fig. 10b. As opposed to the unrestrained simulations, $H_{\text {wat-wat }}$ increases with dehydration as well. The increase in $S_{\text {lip }}=S_{\text {ter }}+S_{\text {tra }}$ presumably arises from the increase in $S_{\text {tra }}$ due to the decrease in the ordering of the lipid tails as the bilayer is stretched.

\section{Polarizable water model}

The effect of explicit polarization of water on the intermembrane repulsion was tested by conducting simulations with the 


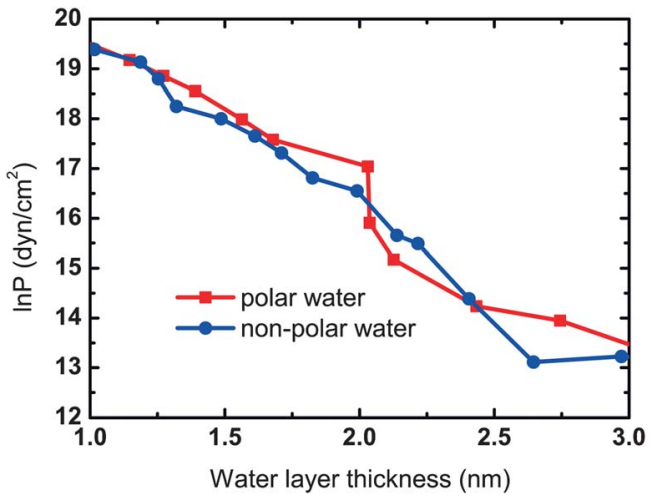

Fig. 11 Disjoining pressure versus water layer thickness for the polar (squares) and the non-polar (circles) water model in semilogarithmic presentation. For an error estimate, see Fig. 6.

polarizable MARTINI water model. ${ }^{47}$ In this model a coarsegrained solvent particle, representing four water molecules, is comprised of three beads, two of which have equal charges of opposite sign. This water has rotational degrees of freedom and a dipole moment, and therefore is similar to atomistic water. The number of lipid and polar water molecules is the same as used for the small POPC system with different hydration levels. The pressure versus distance is shown in Fig. 11, together with the pressure profile for the non-polar water model (small system size). The difference between these profiles is small, demonstrating that both water models are equivalent. However, the polar water model provides additional information about the polarization of water close to the bilayer surface. The average cosine of the angle between the water dipole moment and the bilayer normal is shown in Fig. 12. Bulk polar water has no preferred orientation of the dipole moment, whereas at the interface there is a tendency of water dipoles to orient antiparallel to the bilayer normal with an average angle of about $100^{\circ}$. This orientation is opposite to the atomistic water orientation observed in ref. 24. In the CG model the water orientation is purely due to the dielectric response to the charge distribution from the lipids. In a more realistic atomistic model, on the
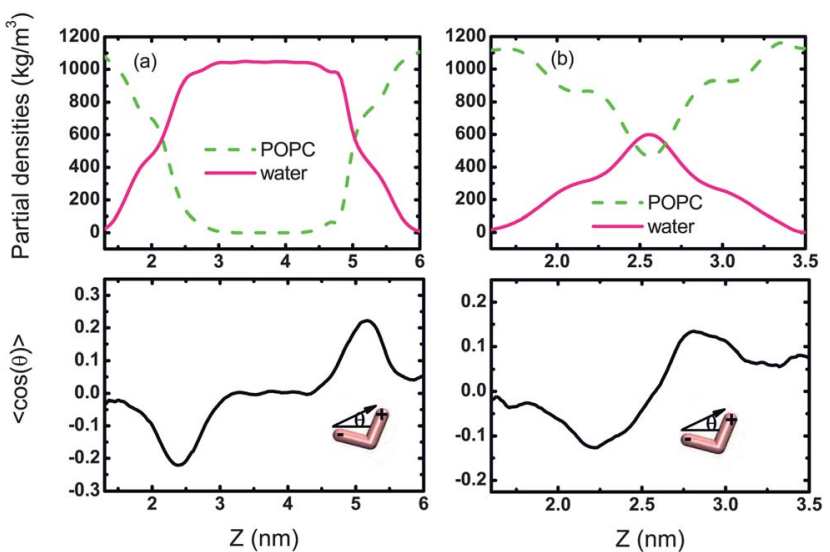

Fig. 12 Partial densities and water dipole orientation profiles near the bilayer interface for (a) 37.5 and (b) 5 water molecules per lipid.

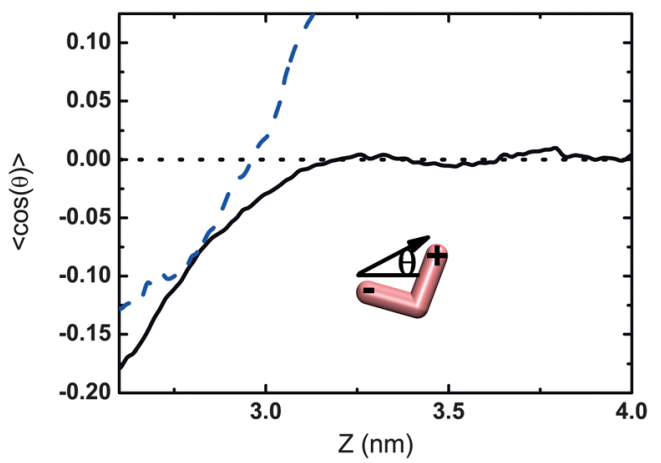

Fig. 13 Comparison of water dipole orientation for two systems with 37.5 (full line) and 5 (dashed line) water molecules per lipid. For the dashed curve the $Z$ coordinate is shifted by $0.38 \mathrm{~nm}$ to bring the two curves closer for comparison.

other hand, the main contribution of the water ordering comes from the interaction of the quadrupole moment with the gradient in the dielectric permeability at the interface, leading to anisotropic water dipole orientation that even overcompensates the contribution of the lipids to the electrostatic potential in the interior of the bilayer. ${ }^{62,63}$ This electrostatic potential arising from the lipids and water is referred to as dipole potential. When only a few molecules are left, all water molecules are bound to the interface and the dipole orientation smoothly changes from $100^{\circ}$ to $80^{\circ}$, with some depolarization effect in the middle of the water slab, as discussed in ref. 24 and shown in Fig. 13. The discrepancy between the atomistic water and the polarizable MARTINI model arises from the interplay between packing (steric) effects and orientation of the water's dipole moment. The packing effects in the coarse-grained and the atomistic model differ significantly.

\section{Conclusions}

Here we have introduced an approach to calculate the disjoining repulsion pressure using the fact that the area and the thickness of the bilayer without restraint change with dehydration in simulations. We based our argumentation on a thermodynamic approach and derived equations to estimate the repulsion pressure from the change in the area per lipid upon dehydration. We compare this approach with an umbrella sampling setup, where the center-of-mass distance between two punctured membranes in a water reservoir is controlled. The model employed is the MARTINI coarse-grained force-field. We demonstrate that this force field reproduces the short-range repulsion and the decay length for POPC lipids obtained from our experimental approach. The balance of interactions depends on the boundary conditions and differs between the unrestrained and the umbrella sampling setup.

Most strikingly, we show that dehydration leads to a decrease in the entropy of the lipids without restraint but an increase in the entropy of the lipids for the umbrella sampling setup. The entropy decrease of the lipids without restraint is mainly attributed to the increase in the ordering of the lipid tails associated with the decrease in the area per lipid, whereas the entropy increase of the lipids for the umbrella sampling setup 
may mainly arise from the decrease in the ordering of the lipid tails due to the stretching of the bilayer (increase in the area per lipid) upon dehydration. The behavior of the area per lipid in the two different setups results from the tendency of the system to minimize its free energy under the given boundary conditions. The balance of interactions obtained from a thermodynamic analysis is strongly determined by the boundary conditions. Changing them may even invert the balance between lipid entropy and other interactions. In this regard, this balance is largely a consequence, rather than a cause, of the intermembrane repulsion. Hence, care must be taken in the interpretation of thermodynamic data in terms of the mechanism underlying the disjoining pressure between membranes.

The good agreement of our calculations with experiments opens the perspective for using our approach to solve related questions, such as to investigate how the intermembrane repulsion can be modulated by the lipid composition as well as the presence of proteins, organic solvents, etc., which change the membrane structure.

\section{Appendix}

Here the equation for the disjoining pressure at a controlled water content is derived. At a given water layer thickness $d_{\mathrm{w}}$ and the corresponding bilayer area $A$ (note that $\left.A=N_{\text {lip }} /\left(L_{z}-d_{\mathrm{w}}\right) \rho_{\mathrm{hh}}\right)$ the excess free energy is minimized with respect to $d_{\mathrm{w}}$. Taking into account the condition of equal pressure components yields the equations

$$
\frac{\partial \Delta F}{\partial d_{\mathrm{w}}}=0
$$

and

$$
P_{L}=P_{N}=0
$$

Using eqn (12) this leads to

$$
A^{\prime}\left(K_{A} \frac{\Delta A}{A_{0}}+g\left(d_{\mathrm{w}}\right)+\frac{\Delta V^{\mathrm{w}}}{\kappa V_{0}^{\mathrm{w}}} d_{\mathrm{w}}\right)+A\left(g^{\prime}\left(d_{\mathrm{w}}\right)+\frac{\Delta V^{\mathrm{w}}}{\kappa V_{0}^{\mathrm{w}}}\right)=0
$$

and

$$
\frac{K_{A}}{L_{z}} \frac{\Delta A}{A_{0}}+\frac{g\left(d_{\mathrm{w}}\right)}{L_{z}}+g^{\prime}\left(d_{\mathrm{w}}\right)\left(1-\frac{d_{\mathrm{w}}}{L_{z}}\right)+\frac{\Delta V^{\mathrm{w}}}{\kappa V_{0}^{\mathrm{w}}}=\frac{\Delta V^{\mathrm{w}}}{\kappa V_{0}^{\mathrm{w}}}+g^{\prime}\left(d_{\mathrm{w}}\right)=0,
$$

where $A^{\prime} \equiv \frac{\partial A}{\partial d_{\mathrm{w}}}=\frac{A}{L_{z}-d_{\mathrm{w}}}$. Therefore the equations reduce to the following first-order linear differential equation

$$
\frac{\mathrm{d} g\left(d_{\mathrm{w}}\right)}{\mathrm{d} d_{\mathrm{w}}}-\frac{g\left(d_{\mathrm{w}}\right)}{d_{\mathrm{w}}}-\frac{K_{A}\left(A-A_{0}\right)}{d_{\mathrm{w}} A_{0}}=0 .
$$

The solution for the first derivative of $g\left(d_{\mathrm{w}}\right)$ is

$$
P\left(d_{\mathrm{w}}\right) \equiv-\frac{\mathrm{d} g\left(d_{\mathrm{w}}\right)}{\mathrm{d} d_{w}}=\frac{K_{A}}{d_{\mathrm{w}}}\left(1-\frac{A}{A_{0}}\right)+\int_{\infty}^{d_{\mathrm{w}}} \frac{K_{A}}{d_{\mathrm{w}}^{\prime 2}}\left(1-\frac{A}{A_{0}}\right) \mathrm{d} d_{w}^{\prime} .
$$

Here $P\left(d_{\mathrm{w}}\right)$ is the disjoining pressure at bilayer separation space $d_{\mathrm{w}}$ (the intermembrane repulsion is zero at infinitely large distances). The contribution from the integral term is small (because the pressure decays fast with the distance) and can be neglected. Note that ref. 25 does not show the integral contribution for the disjoining pressure because the interface potential was calculated for each lipid. With this convention the integral in eqn (23) will vanish.

Moreover we show that in the canonical ensemble the disjoining pressure equals the osmotic pressure. The chemical potential of water is $\mu=\frac{\partial F}{\partial N_{\mathrm{w}}}$ which leads to

$$
\frac{\Delta \mu}{v_{\mathrm{w}}}=\frac{K_{A}}{d_{\mathrm{w}}} \frac{\Delta A}{A_{0}}+g^{\prime}\left(d_{\mathrm{w}}\right)+\frac{g\left(d_{\mathrm{w}}\right)}{d_{\mathrm{w}}}+\frac{\Delta V^{\mathrm{w}}}{\kappa V_{0}^{\mathrm{w}}},
$$

where $v_{\mathrm{w}}$ is the partial volume of water molecules. Using eqn (21) and (22) yields

$$
\frac{\Delta \mu}{v_{\mathrm{w}}}=g^{\prime}\left(d_{\mathrm{w}}\right)=-P\left(d_{\mathrm{w}}\right) .
$$

\section{Acknowledgements}

We thank R. Lipowsky, M. Kozlov, G. Marelli, D. Bedrov, and V. Baulin for stimulating discussions and the SFB-803 and VWfoundation for financial support. We acknowledge the computational facilities of the MPIKG computer cluster, supercomputer centers HLRN Hannnover/Berlin and NIC Jülich.

\section{References}

1 T. J. McIntosh and S. A. Simon, Annu. Rev. Biophys. Biomol. Struct., 1994, 23, 27.

2 R. Lipowsky, in Handbook of Biological Physics, ed. R. Lipowsky and E. Sackmann, Elsevier, 1995, vol I, p. 521.

3 G. Brannigan and F. Brown, Biophys. J., 2006, 90, 1501.

4 R. I. MacDonald, Biochemistry, 1985, 24, 4058.

5 S. Leikin, V. A. Parsegian, D. C. Rau and R. P. Rand, Annu. Rev. Phys. Chem., 1993, 44, 369.

6 V. Varma, M. Taalbi, J. M. Collins, W. Tamura-Lis and L. J. Lis, J. Colloid Interface Sci., 1989, 133, 427.

7 J. Marra and J. Israelachvili, Biochemistry, 1985, 24, 4608.

8 R. P. Rand, Annu. Rev. Biophys. Bioeng., 1981, 10, 277.

9 S. Marcelja and N. Radic, Chem. Phys. Lett., 1976, 42, 129.

10 J. N. Israelachvili and H. Wennerstroem, Langmuir, 1990, 6, 873.

11 R. Podgornik and V. A. Parsegian, Langmuir, 1992, 8, 557. 12 R. Lipowsky and S. Grotehans, Europhys. Lett., 1993, 23, 599. 13 R. Lipowsky and S. Grotehans, Biophys. Chem., 1994, 49, 27. 14 L. Perera, U. Essmann and M. L. Berkowitz, Langmuir, 1996, $12,2625$.

15 A. Perstin, D. Platonov and M. Grunze, J. Chem. Phys., 2005, 122, 244708.

16 A. Perstin, D. Platonov and M. Grunze, Langmuir, 2007, 23, 1388.

17 C. Eun and M. L. Berkowitz, J. Phys. Chem. B, 2009, 113, 13222.

18 C. Eun and M. L. Berkowitz, J. Phys. Chem. B, 2010, 114, 3013. 19 A. N. Gentilcore, N. Michaud-Agrawal, P. S. Crozier, M. J. Stevens and T. B. Woolf, J. Membr. Biol., 2010, 235, 1. 20 K. Hristova and S. H. White, Biophys. J., 1998, 74, 2419. 
21 S. Aeffner, T. Reusch, B. Weinhausen and T. Salditt, Proc. Natl. Acad. Sci. U. S. A., 2012, 109, E1609.

22 V. Knecht and B. Klasczyk, Biophys. J., 2013, 104, 818.

23 E. Schneck and R. R. Netz, Curr. Opin. Colloid Interface Sci., 2011, 16, 607.

24 E. Schneck, F. Sedlmeier and R. R. Netz, Proc. Natl. Acad. Sci. U. S. A., 2012, 109, 14405.

25 V. A. Parsegian, N. Fuller and R. P. Rand, Proc. Natl. Acad. Sci. U. S. A., 1979, 76, 2750.

26 E. A. Golovina, A. V. Golovin, F. A. Hoekstra and R. Faller, Biophys. J., 2009, 97, 490.

27 S. W. Chiu, M. Clark, V. Balaji, S. Subramaniam, H. L. Scott and E. Jakobsson, Biophys. J., 1995, 69, 1230.

28 R. Goetz and R. Lipowsky, J. Chem. Phys., 1998, 108, 7397.

29 S. J. Marrink, H. J. Risselada, S. Yefimov, D. P. Tieleman and A. H. de Vries, J. Phys. Chem. B, 2007, 111, 7812.

30 S. J. Marrink and A. E. Mark, J. Am. Chem. Soc., 2003, 125, 11144.

31 Y. G. Smirnova, S. J. Marrink, R. Lipowsky and V. Knecht, J. Am. Chem. Soc., 2010, 132, 6710.

$32 \mathrm{H}$. J. Risselada, C. Kutzner and H. Grubmüller, ChemBioChem, 2011, 12, 1049.

33 H. J. Risselada, G. Marelli, M. Fuhrmans, Y. G, Smirnova, H. Grubmüller, S. J. Marrink and M. Müller, PLoS One, 2012, 7, e38302.

34 L. J. Lis, M. McAllister, N. Fuller, R. P. Rand and V. A. Parsegian, Biophys. J., 1982, 37, 657.

35 R. P. Rand and V. A. Parsegian, Biochim. Biophys. Acta, 1989, 988, 351.

36 J. F. Nagle and S. Tristram-Nagle, Biochim. Biophys. Acta, 2000, 1469, 159.

37 V. A. Parsegian, R. P. Rand, N. L. Fuller and D. C. Rau, Methods Enzymol., 1986, 127, 400.

38 S. K. Ghosh, S. Aeffner and T. Salditt, ChemPhysChem, 2011, 12, 2633.

39 S. Aeffner, T. Reusch, B. Weinhausen and T. Salditt, Eur. Phys. J. E, 2009, 30, 205.

40 D. V. Lynch and P. L. Steponkus, Biochim. Biophys. Acta, 1989, 984, 267.
41 S. Aeffner, Stalk Structures in Lipid Bilayer Fusion Studied by X-ray Diffraction, Ph.D. thesis, University of Göttingen, 2011. 42 D. van der Spoel, E. Lindahl, B. Hess, G. Groenhof, A. E. Mark and H. J. C. Berendsen, J. Comput. Chem., 2005, 26, 1701.

43 H. J. C. Berendsen, J. P. M. Postma, W. F. van Gunsteren, A. DiNola and J. R. Haak, J. Chem. Phys., 1984, 81, 3684.

44 H. J. Risselada, A. E. Mark and S. J. Marrink, J. Phys. Chem. B, 2008, 112, 7438.

45 G. M. Torrie and J. P. Valleau, J. Comput. Phys., 1977, 23, 187. 46 S. Kumar, J. M. Rosenberg, D. Bouzida, R. H Swendsen and P. A. Kollman, J. Comput. Chem., 1992, 13, 1011.

47 S. O. Yesylevskyy, L. V. Schäfer, D. Sengupta and S. J. Marrink, PLoS Comput. Biol., 2010, 6, e1000810.

48 J. A. McNew, T. Weber, F. Parlati, R. J. Johnston, T. J. Melia, T. H. Söllner and J. E. Rothman, J. Cell Biol., 2000, 150, 105. 49 V. Knecht and H. Grubmüller, Biophys. J., 2003, 84, 1527.

50 H. Binder and K. Gawrisch, J. Phys. Chem. B, 2001, 105, 12378.

51 W. Rawicz, K. C. Olbrich, T. McIntosh, D. Needham and E. Evans, Biophys. J., 2000, 79, 328.

52 D. Poger and A. E. Mark, J. Chem. Theory Comput., 2010, 6, 325.

53 Z. J. Wang and M. Deserno, J. Phys. Chem. B, 2010, 144, 11207.

54 M. Hömberg and M. Müller, J. Chem. Phys., 2010, 132, 155104.

55 C. von Deuster and V. Knecht, Biochim. Biophys. Acta, Biomembr., 2012, 1818, 2192.

56 S. Nosé, Mol. Phys., 1984, 52, 255.

57 W. Hoover, Phys. Rev. A: At., Mol., Opt. Phys., 1985, 31, 1695.

58 M. Parrinello and A. Rahman, J. Appl. Phys., 1981, 52, 7182.

59 N. Kučerka, S. Tristram-Nagle and J. F. Nagle, J. Membr. Biol., 2005, 208, 193.

60 F. Sedlmeier, D. Horinek and R. R. Netz, J. Am. Chem. Soc., 2011, 133, 1391.

61 S. Sarupria and S. Garde, Phys. Rev. Lett., 2009, 103, 037803.

62 R. A. Böckmann, A. Hac, T. Heimburg and H. Grubmüller, Biophys. J., 2003, 85, 1647.

63 C. A. Croxton, Physica A, 1981, 106, 239. 